\section{MedienPädagogik}

www.medienpaed.com
Zeitschrift für

Theorie und Praxis

der Medienbildung

ISSN 1424-3636

Themenheft Nr. 21: Partizipationschancen im Kulturraum Internet nutzen und gestalten - Das Beispiel Web 2.0

\title{
Politische Bildung und das Web 2.0
}

Heike Schaumburg

\begin{abstract}
Aktuell erhält die Diskussion um die Rolle des Internet in der Politischen Bildung neuen Auftrieb durch die Entwicklung der sogenannten Web-2.0-Technologien, die im Vergleich zum Web 1.0 ein erheblich grösseres Potenzial zur Mitgestaltung politischer Inhalte und Prozesse und bzw. zur Partizipation bieten, aber auch neue Möglichkeiten der Kommunikation und Vernetzung eröffnen.

In der vorliegenden Studie wird der Frage nachgegangen, inwieweit das Internet und speziell das Web 2.0 in der Lage sind, die in die Technik gesetzten Hoffnungen zu erfüllen. Dabei wird zunächst ausgelotet, welche Potenziale das Web 2.0 für die Politische Bildung mit Blick auf ihre didaktische Gestaltung bieten könnte. Im Anschluss daran wird auf der Basis einer Analyse des Internet-Angebots der traditionellen Anbieter Politischer Bildung (Bundes- und Landeszentralen für Politische Bildung, politische Stiftungen) sowie von Internetportalen im Bereich Politische Bildung festgestellt, welche Verbreitung Web-2.0-Angebote gegenwärtig besitzen. Schliesslich wird auf der Grundlage einer Nutzerbefragung an knapp 300 Teilnehmern virtueller politischer Bildungsangebote einer politischen Stiftung der Frage nachgegangen, wie Web-2.0-Angebote in der Politischen Bildungsarbeit aus Nutzersicht beurteilt werden.
\end{abstract}

\section{Potenziale des Web 2.0 für die Politische Bildung}

In didaktischer Hinsicht weisen die Eigenschaften des Web 2.0 eine hohe Passung zu den Besonderheiten der Politischen Bildung auf, die nach Wache (2003) und Harth (2000) wie folgt zusammengefasst werden können:

- In der Didaktik der Politischen Bildung spielen kommunikative Lernformen, wie Diskussion, Streitgespräch, Erfahrungsaustausch oder Projektkooperation eine herausragende Rolle.

- Politische Bildung zielt neben der Vermittlung von Wissen und Fertigkeiten auch und gerade auf die Gestaltung von Einstellungen, Wertorientierungen, Emotionen und Interessen ab.

- Die zu vermittelnden Inhalte, d. h. politische Zusammenhänge und Wirkungen sind beinahe grundsätzlich hochkomplex. 
Eine herausragende Eigenschaft von Web-2.0-Applikationen (wie z. B. Wikis und Weblogs) besteht darin, dass sie prinzipiell jedem Internetnutzer die Möglichkeit eröffnen, Informationen öffentlich zu erstellen, zu verändern oder zumindest zu kommentieren. Anwendungen des Web 2.0 bieten somit neue Möglichkeiten, Inhalte über das Netz mit anderen Personen gemeinsam zu erarbeiten, Meinungen auszutauschen und Kontakte zu knüpfen. Dies gilt nicht allein für den E-LearningKontext, sondern kennzeichnet einen grundsätzlichen Wandel in den Interaktionsund Partizipationsmöglichkeiten, die sich über das Internet (auch) für die Teilhabe an gesellschaftlichen und politischen Prozessen ergeben. Die Beschäftigung mit dem Web 2.0 ist somit für die Politische Bildung nicht nur aus formalen, sondern auch aus inhaltlichen Gründen von grossem Interesse.

Als Werkzeuge des politischen Diskurses im Internet werden schon seit langem Online-Diskussionsforen in der Politischen Bildung eingesetzt. Diese können als einer der Vorläufer der heutigen Web-2.0-Technologien gelten. Das Potenzial solcher elektronischer Foren im Vergleich zu Diskussionen in Präsenzveranstaltungen kann darin gesehen werden, dass die Diskussion weniger «flüchtig» ist als eine Präsenzveranstaltung. Dies gibt den Teilnehmenden mehr Zeit, eigene Beiträge zu formulieren und sich mit Diskussionsbeiträgen anderer auseinander zu setzen. Davon sollten vor allem Teilnehmern profitieren, die sich scheuen, spontan in einer Gruppe zu sprechen. Weiterhin hofft man, dass die asynchrone Bearbeitung, die den Lernenden mehr Zeit für die Reaktion auf einen Beitrag einräumt sowie die Verschriftlichung der Beiträge zu einer intensiveren Auseinandersetzung mit fremden Positionen und der eigenen führt als die mündliche Diskussion (Jelich, 2003). Der Fokus auf Einstellungen, Wertorientierung und Emotionen in der Politischen Bildung wird adressiert, wenn Blogs als Medium politischer Artikulation genutzt werden (Rosa, 2006; Ott, 2006). Ein zentrales Merkmal dieser Kommunikationsform ist, dass ihr Verfasser sich in einer sehr subjektiven, persönlichen Form zu (politischen) Themen äussert. Als Medium politischer Bildung wird das Potenzial von Blogs z. B. darin gesehen, dass beim Schreiben eines Blogs politische Sachverhalte reflektiert, aber eben auch explizit bewertet werden müssen. Auch das Lesen von Blogs kann der Reflexion unterschiedlicher Werthaltungen und Einstellungen dienen (Rosa, 2006).

Mit Bezug auf die kooperative Wissenskonstruktion werden weiterhin die Möglichkeiten von Wikis in der Politischen Bildung bzw. genereller als Medium des politischen Diskurses ausgelotet (z. B. Brombach, 2007; Westermeyer, 2007). Brombach (2007) sieht didaktische Vorteile darin, dass die gemeinsame Arbeit an einem Wiki zwangsläufig zur Auseinandersetzung mit den Perspektiven der Co-Autoren führt, da Texte möglicherweise individuell erstellt, dann jedoch in jedem Fall kollektiv überarbeitet werden. Wikis werden auch zur Unterstützung komplexen Lernens diskutiert, da die Teilnehmer sich bei der gemeinsamen Erstellung eines Wikis in- 
tensiv mit der Struktur eines politischen Themas, seiner Zusammenhänge und Abhängigkeiten beschäftigen müssen (Brombach, 2007).

Ebenfalls auf politische Einstellungen, Werte und letztendlich zivilgesellschaftliches Engagement zielt die Politische Bildungsarbeit mit virtuellen Communitys ab, die vor allem im US-amerikanischen Raum fortgeschritten ist. Solche Plattformen stellen eine Vielzahl von Werkzeugen bereit, z. B. Blogs, Profilseiten, über die die Mitglieder ihre Interessen darstellen und sich miteinander vernetzen können, Web Messenger, Diskussionsforen, Verzeichnisse und Datenbanken von politischen Organisationen und Aktionen (die auch von den Nutzern erweitert werden können), Fotogalerien, Werkzeuge für kreatives Schreiben, usw. (Raynes-Goldie \& Walker, 2008). Die Idee hierbei ist weniger die durch einen Anbieter gesteuerte Vermittlung politischer Inhalte, als die, eine Plattform zu schaffen, die politische Interessierten Möglichkeiten bietet, sich mit Gleichgesinnten zu vernetzen, um im und ausserhalb des Internet politisch aktiv zu werden. In didaktischer Hinsicht besteht der Mehrwert solcher Plattformen gegenüber traditionellen politischen Bildungsangeboten darin, dass der politische Lernprozess hier konsequent in die Hand der Lernenden gelegt und eine - sofern es um reales politisches Engagement geht - wirklich authentische Lernsituation geschaffen wird. In einer solchen Umgebung tritt an die Stelle des Lehrer-Lerner-Dualismus eine lernende Gemeinschaft aus gleichberechtigten Individuen - der Gedanke des Web 2.0 wird hier am umfassendsten realisiert. Gleichzeitig lässt sich fragen, ob solche Communitys für sich genommen überhaupt noch als Angebote Politischer Bildung betrachtet werden können, da sie in der Regel kaum strukturierte und didaktisierte Lernangebote aufweisen sondern primär informelles Lernen unterstützen (Ehlers, 2008).

Tab. 1 veranschaulicht die Potenziale des Web 2.0 für die Politische Bildung im Überblick.

\begin{tabular}{|l|l|l|}
\hline Besonderheiten politischer Bildung & Eigenschaften des Web 2.0 & Werkzeug-Beispiele \\
\hline $\begin{array}{l}\text { Gestaltung von Einstellungen, } \\
\begin{array}{l}\text { Wertorientierungen, Emotionen und } \\
\text { Interessen }\end{array}\end{array}$ & $\begin{array}{l}\text { Nutzer als Produzent, Spontaneität, } \\
\text { Subjektivität, nicht-hierarchische } \\
\text { Kommunikation, Vernetzung und } \\
\text { Community-Bildung }\end{array}$ & $\begin{array}{l}\text { Blog, Online-Forum, } \\
\text { Social Community }\end{array}$ \\
\hline $\begin{array}{l}\text { Besondere Bedeutung kommunika- } \\
\text { tiver Lernformen (Diskussion, Streit- } \\
\text { gespräch, Erfahrungsaustausch, } \\
\text { Projektkooperation) }\end{array}$ & $\begin{array}{l}\text { Nutzer als Produzent, Spontaneität, } \\
\text { Sommunikation } \\
\text { Komivät, nicht-hierarchische }\end{array}$ & $\begin{array}{l}\text { Blog, Online-Forum, Wiki, } \\
\text { Social Community }\end{array}$ \\
\hline $\begin{array}{l}\text { Hochkomplexe Inhalte (politische } \\
\text { Systeme, Zusammenhänge, Wir- } \\
\text { kungen) }\end{array}$ & $\begin{array}{l}\text { Ko-operatives Erarbeiten und Dar- } \\
\text { stellen komplex vernetzter Inhalte, } \\
\text { kontinuierliche Überarbeitungs- } \\
\text { und Veränderungsmöglichkeiten }\end{array}$ & Wiki \\
\hline
\end{tabular}

Tab. 1.: Potenziale des Web 2.0 für die Politische Bildung 


\section{Angebotsanalyse: Web 2.0 in der internetbasierten Politischen Bildung}

Von Berichten zu einzelnen Pilotprojekten abgesehen (z. B. Brombach, 2007; Jelich, 2003; Fuchs, 2006) gibt es bisher kaum Aussagen dazu, welche Verbreitung Web-2.0-Angebote in der Politischen Bildung zurzeit besitzen. Im Rahmen der vorliegenden Untersuchung wurde deshalb eine Angebotsanalyse durchgeführt, bei der die Webseiten verschiedener deutscher Anbieter Politischer Bildung systematisch nach internetbasierten Bildungsangeboten durchsucht wurden. In die Analyse wurden die Angebote der politischen Stiftungen sowie der Bundeszentrale und der Landeszentralen für Politische Bildung einbezogen. Darüber hinaus wurde nach Angeboten von auf das Internet spezialisierten Anbietern Politischer Bildung gesucht, die über Portalseiten der Bundeszentrale sowie durch SuchmaschinenRecherche, die auch deutschsprachige Angebote ausländischer politischer Bildungsinstitutionen (Österreich, Schweiz) einschloss, recherchiert wurden. Unter den Internet-Anbietern sind nur solche in die Analyse aufgenommen worden, die ein breites Spektrum gesellschaftspolitischer Fragen thematisieren. Insofern bilden insbesondere die analysierten Websites der Internet-Anbieter nur einen kleinen Ausschnitt einer wesentlich grösseren Anzahl von Plattformen spezieller Interessengruppen, die Möglichkeiten zur Diskussion und Vernetzung zu politischen Einzelthemen bereitstellen.

Für alle Anbieter wurde zum einen erfasst, inwiefern sie klassische E-Learning-Angebote, d. h. betreute und unbetreute Online-Kurse und Selbstlernangebote machen, zum anderen, ob und in welchem Umfang sie Technologien einsetzen, die sich im weitesten Sinne dem Web 2.0 zurechnen lassen (Diskussionsforen, Blogs, Wikis und Online Communitys). Tabelle 2 zeigt, welche Angebotsformen bei welchen Anbietern gefunden wurden.

Die Übersicht lässt deutlich werden, dass das Web 2.0 klassischen E-Learning-Angeboten in der internetbasierten Politischen Bildung bereits den Rang abgelaufen hat. Insgesamt sind besonders Diskussionsforen und Blogs auf den Internetseiten politischer Bildungsanbieter inzwischen recht verbreitet. Angebote, die in Richtung einer Online-Community gehen, sind ebenfalls im Entstehen. Lediglich der Einsatz von Wikis stellt in der Politischen Bildung bisher eher eine Ausnahme dar. Die Zielgruppen und die Gestaltung der Angebote ist breit gefächert: So werden Blogs und Foren innerhalb didaktisch aufbereiteter Online-Kurse (z. B. politische Stiftungen, Landeszentralen) oder zur Vor- und Nachbereitung von Präsenzveranstaltungen eingesetzt (Friedrich-Ebert-Stiftung). Weiterhin werden sie als offenes Angebot genutzt, über das die Netzöffentlichkeit sich zu beständig aktualisierten politischen Themen äussern kann (Landeszentrale Brandenburg, Bundeszentrale, diverse Internetanbieter). Einige Anbieter sehen über Foren und Blogs hinausgehende Angebote vor, z.B. die Möglichkeiten, Teilnehmerprofilseiten anzulegen, Teilnehmer direkt über die Plattform zu kontaktieren, Fotoalben zu erstellen, Vi- 


\begin{tabular}{|c|c|c|c|c|c|c|c|}
\hline & 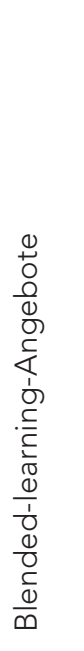 & 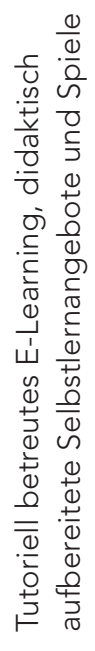 & 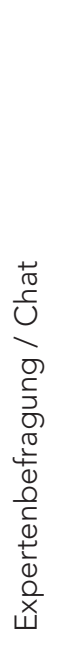 & 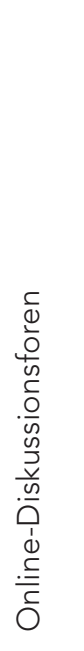 & $\frac{\mathscr{\rho}}{0}$ & $\stackrel{\frac{n}{y}}{s}$ & 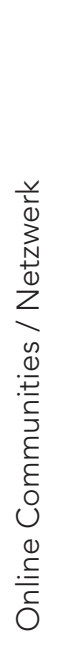 \\
\hline \multicolumn{8}{|l|}{ Politische Stiftungen } \\
\hline \multicolumn{8}{|l|}{ Konrad-Adenauer-Stiftung } \\
\hline Friedrich-Ebert-Stiftung & $x$ & $x$ & & $x$ & & & \\
\hline Heinrich-Böll-Stiftung & & & & & $x$ & & \\
\hline Rosa-Luxemburg-Stiftung & & & & & & $x$ & \\
\hline \multicolumn{8}{|l|}{ Hanns-Seidel-Stiftung } \\
\hline $\begin{array}{l}\text { Friedrich-Naumann-Stiftung für die } \\
\text { Freiheit }\end{array}$ & $x$ & $x$ & $x$ & $x$ & $x$ & $x$ & $x$ \\
\hline \multicolumn{8}{|l|}{ Zentralen für Politische Bildung } \\
\hline Bundeszentrale für Politische Bildung & & $x$ & & $x$ & $x$ & & $x$ \\
\hline Landeszentrale Bayern & & $x$ & & & & & $x$ \\
\hline Landeszentrale Baden-Württemberg & $x$ & $x$ & & & & & \\
\hline Landeszentrale Brandenburg & & & & $x$ & $x$ & & \\
\hline Landeszentrale Nordrhein-Westfalen & & $x$ & & & & & \\
\hline Landeszentrale Rheinland-Pfalz & $x$ & $x$ & & & & & \\
\hline Landeszentrale Sachsen & & $x$ & & & & & \\
\hline $\begin{array}{l}\text { Landeszentralen Berlin, Bremen, } \\
\text { Hamburg, Hessen, Mecklenburg-Vor- } \\
\text { pommern, Saarland, Sachsen-Anhalt, } \\
\text { Schleswig-Holsten, Thüringen }\end{array}$ & & & & & & & \\
\hline
\end{tabular}




\begin{tabular}{|c|c|c|c|c|c|c|}
\hline \multicolumn{7}{|l|}{ Sonstige Internetangebote } \\
\hline Aktion Mensch: Die Gesellschafter & & & $x$ & $x$ & & $x$ \\
\hline www.politik.de & $x$ & $x$ & $x$ & $x$ & & $x$ \\
\hline www.politik-digital.de & & $x$ & & $x$ & & \\
\hline www.politikforum.com & & & $x$ & $x$ & & \\
\hline www.politik-forum.de & & & $x$ & & & \\
\hline www.politik-foren.net & & & $x$ & & & \\
\hline www.dol2day.com & & $x$ & $x$ & $x$ & & $x$ \\
\hline www.vdeutschland.de & & & $x$ & & & $x$ \\
\hline www.polipedia.at & & & & $x$ & $x$ & \\
\hline www.polittalk.ch & & & $x$ & & & $x$ \\
\hline
\end{tabular}

Tab. 2: Internetangebote verschiedener Träger politischer Bildung

deos hochzuladen oder virtuelle Gruppen zu bilden (z. B. Friedrich-NaumannStiftung für die Freiheit, Bundeszentrale, www.politik.de, www.dol2day.com, www. polittalk.ch). Solche Angebote dienen der Vernetzung von Teilnehmern virtueller Lernangebote, bzw. der langfristigen Kontaktsicherung für Ehemalige (z. B. Friedrich-Naumann-Stiftung für die Freiheit) oder der Bekanntmachung und Vernetzung von Gruppen und Initiativen (z. B. Landeszentrale Bayern, Die Gesellschafter), bzw. der Bildung von Interessengruppen zu politischen Themen (www.politik.de, www. polittalk.ch). Sogenannte «Micro-Nations» wie dol2day und vDeutschland sind internetbasierte Plattformen, mit denen politische Prozesse bzw. demokratische Systeme simuliert und damit für die Nutzer spielerisch erfahrbar werden. So können Nutzer hier politische Parteien gründen, Mitglieder für die Parteien werben und sich an virtuellen Wahlen beteiligen, indem sie selbst wählen oder sich zur Wahl stellen. Die Inhalte, die auf der Plattform verhandelt werden, sind dabei in der Regel nicht plattform-intern und fiktiv, sondern orientieren sich an aktuellen gesellschaftspolitischen Debatten und Themen, die die Mitglieder der Plattform zur Diskussion und Abstimmung stellen.

Betrachtet man die verschiedenen Anbieter-Gruppen, so fallen deutliche Unterschiede in der Angebotsstruktur auf: Die politischen Stiftungen machen (mit Ausnahme der Friedrich-Naumann-Stiftung für die Freiheit) bisher nur vereinzelte internet-basierte Lernangebote (und zwar sowohl im «traditionellen» E-Learning wie auch bezogen auf Web-2.0-Anwendungen). Sie nutzen das Internet vor allem, um über die Stiftung und ihr Angebot zu informieren, bzw. im Bereich der Politischen Bildung ihre Präsenzveranstaltungen zu bewerben und zu dokumentieren. Ähnliches gilt auch für die Mehrheit der Landeszentralen. Wird das Internet von diesen 
als Lernmedium genutzt, fällt auf, dass diese stark dem herkömmlichen E-Learning verpflichtet sind. So verfügen zahlreiche Landeszentralen über Selbstlernangebote, Quizze und Spiele sowie vereinzelt auch über betreute E-Learning-Kurse. Auch die Bundeszentrale hält ein breites und technisch sowie didaktisch aufwändig gestaltetes Angebot zum unbetreuten eigenverantwortlichen Lernen vor. Werkzeuge für den öffentlichen politischen Diskurs und Meinungsaustausch bietet die Bundeszentrale für Politische Bildung lediglich in ihrem Jugendbereich an.

Genau entgegengesetzt verhält es sich mit den auf das Internet spezialisierten Interessengruppen und Initiativen: Hier bilden Diskussionsforen und Blogs das Kernstück des Angebots. Teilweise werden zu den Diskussionen Experten eingeladen, mit denen im Forum oder mittels Chat kommuniziert werden kann. E-LearningKurse oder Selbstlernangebote finden sich dagegen bei diesen Anbietern so gut wie gar nicht. Auch bilden politische Basis- oder Rahmeninformationen, wenn es solche überhaupt gibt, lediglich den Aufhänger für die Diskussionen, bzw. sind solche in blogspezifischer Art als relativ knapper persönlicher Kommentar verfasst. Im Vergleich zum Angebot der Zentralen und Stiftungen sind politische Grundlagen bei diesen Anbietern meistens weniger umfangreich und fundiert sowie kaum didaktisiert.

Zusammenfassend lässt sich feststellen, dass sich mit dem Web 2.0 die Nutzung des Internet in der Politischen Bildung zu wandeln scheint. Während noch vor einigen Jahren von Ruprecht (2002) und Wache (2003) ein Defizit an E-LearningAngeboten, die die technischen Interaktions- und Kommunikationsmöglichkeiten ausschöpfen, konstatiert wurde, so lassen sich inzwischen zahlreiche Beispiele finden, die gerade die Kommunikations- und Vernetzungsmöglichkeiten des Internet in vielfältiger Hinsicht nutzen. Allerdings gibt es bisher nur wenige Beispiele, wo es gelingt, die Nutzung dieser Werkzeuge mit einer fundierten didaktischen Aufarbeitung politischer Themen zu verknüpfen. Die «klassischen» Anbieter politischer Bildung partizipieren bisher eher wenig an der Hinwendung zum Web 2.0 und die Angebote, die sie machen (z. B. Diskussionsforen zur Ergänzungen von Präsenzveranstaltungen) werden eher wenig genutzt. Anbieter von Internet-Communitys mit politischem Inhalt erfreuen sich dagegen teilweise einer sehr regen Teilnahme, besonders wenn sie ihre Seiten in kurzen Abständen aktualisieren, tagespolitische Ereignisse kurzfristig aufgreifen und Experten in die Diskussion einbeziehen, bereiten die politischen Themen jedoch deutlich weniger umfangreich auf. Für die Politische Bildung ist nun die Frage interessant, wie sich Web-2.0-Werkzeuge mit ihren stärker didaktisierten Angeboten verbinden lassen, ob sie sich eher auf virtuelle Diskussionsformate wie in den oben angesprochenen Communitys verlegen oder bei einem klassischen stärker didaktisierten Angebot bleiben sollten. 


\section{Web 2.0 in der Politischen Bildung aus Nutzersicht}

Die Gelegenheit, der Frage nach der Sinnfälligkeit der Einbindung von Web-2.0Angebote in virtuelle Veranstaltungen der Politischen Bildung nachzugehen, bot sich im Rahmen der Evaluation des Internet-Angebots einer politischen Stiftung. Dabei konnte der Einsatz von Web-2.0-Technologien in zwei typischen E-LearningSzenarien vergleichend untersucht werden, da die Stiftung zum Zeitpunkt der Untersuchung verschiedene Veranstaltungsformate anbot: rein virtuell durchgeführte politische Diskussionsseminare, die eine gewisse Nähe zu den oben dargestellten offenen Diskussionsplattformen aufweisen, und Blended-Learning-Veranstaltungen, die eher dem Aufbau von Fertigkeiten und Kompetenzen in der politischen Vereinsarbeit dienen. In beiden Formaten werden Web-2.0-Technologien eingesetzt. Sie unterscheiden sich jedoch in ihren Inhalten und Zielsetzungen sowie bezüglich verschiedener organisatorischer und struktureller Rahmenbedingungen (Format, Dauer, Teilnehmerzahl, Zertifizierung). Wesentliche Merkmale der beiden Kursformate zeigt Tabelle 3.

\begin{tabular}{|l|l|l|}
\cline { 2 - 3 } \multicolumn{1}{l|}{} & Virtuelle politische Diskussion & Blended-Learning-Seminar \\
\hline Lernziel & $\begin{array}{l}\text { Information, Meinungsbildung } \\
\text { zu politischen Themen }\end{array}$ & $\begin{array}{l}\text { Vermittlung von Fertigkeiten für } \\
\text { politische Arbeit }\end{array}$ \\
\hline Format & $\begin{array}{l}\text { ausschliesslich online } \\
\text { Plended Learning (üblicherweise ein } \\
\text { Onlinephasen, die das Präsenztreffen } \\
\text { vor- und nachbereiten) }\end{array}$ \\
\hline $\begin{array}{l}\text { Didruktur } \\
\text { Werkzeuge }\end{array}$ & $\begin{array}{l}\text { betreute Diskussionsphasen, } \\
\text { tw. Lektionen, Einbezug externer Experten } \\
\text { Tests, Arbeitsblätter, Wiki }\end{array}$ & $\begin{array}{l}\text { Lektionen, betreute Bearbeitung } \\
\text { von Aufgaben, Einbezug externer } \\
\text { Experten }\end{array}$ \\
\hline $\begin{array}{l}\text { Teilnehmer- } \\
\text { zahl }\end{array}$ & 2-3 Wochen & $\begin{array}{l}\text { Diskussionsforum, Chat, Materialien, } \\
\text { Blog, Tests, Arbeitsblätter, Wiki }\end{array}$ \\
\hline Zertifizierung & no-200 & $3-6$ Wochen \\
\hline
\end{tabular}

Tab. 3: Untersuchte Kursformate

\section{Methode}

Im September des Jahres 2008 wurde unter allen in diesem Jahr im Online-Bereich der Stiftung angemeldeten Nutzern eine Online-Befragung durchgeführt. Von den 1.341 angeschriebenen Personen beteiligten sich insgesamt 274 Personen an der Befragung. 214 von diesen hatten im Erhebungsjahr zuletzt an einer rein virtuell 
durchgeführten politischen Diskussionsveranstaltung teilgenommen, die anderen 60 Teilnehmer hatten zuletzt ein Blended-Learning-Seminar besucht. Die Beteiligungsquote lag für die politischen Diskussionsseminare bei $20 \%$, für die BlendedLearning-Seminare bei 24\%. Repräsentativitätsanalysen der Stichprobe in Bezug auf die Geschlechts- als auch auf die Altersverteilung anhand der Daten Grundgesamtheit aller im Online-Bereich der Stiftung eingeschriebenen Teilnehmer im Zeitraum 2002 bis 2008 ergaben Konkordanzraten von über $90 \%$.

Inhalte der Befragung waren unter anderem die Bewertung der Gestaltung und Organisation des zuletzt besuchten Seminars und der verwendeten Werkzeuge. Die Beantwortung der Items erfolgte anhand von fünfstufigen Ratingskalen, welche durch offene Fragen ergänzt wurden.

\section{Ergebnisse}

Insgesamt äussert sich die Mehrheit von 70\% der Befragten positiv zu dem zuletzt besuchten Online-Seminar und beschreibt es mit "gut» oder sogar «sehr gut». Explizit negativ wird das zuletzt besuchte Seminar nur von 7\% der Befragten eingeschätzt. Im Mittel erhielten die Blended-Learning-Seminare auf der verwendeten 5er-Skala eine Bewertung von 1.84, die politische Diskussionen die Bewertung 2.31. Dieser Mittelwertsunterschied ist statistisch sehr signifikant $(t=3.43 ; d f=$ $224 ; p<.01)$.

Um Aufschluss über Gründe für diese unterschiedliche Bewertung zu erhalten, wurde zunächst die Akzeptanz der verwendeten Werkzeuge überprüft. Insgesamt fiel die Bewertung für alle Werkzeuge recht positiv aus. Am besten wurden die Informationsanteile der Seminare (Dokumente \& Links) beurteilt, aber auch die Kommunikations- und Interaktionswerkzeuge erhielten durchweg positive Bewertungen. Blended-Learning- und rein virtuelle Veranstaltungen unterscheiden sich dabei hinsichtlich der Beurteilung der Werkzeuge nicht, in der Tendenz werden die meisten Werkzeuge sogar von den Teilnehmern politischer Diskussionen etwas besser beurteilt (vgl. Abb. 1).

Um abzuschätzen, ob es dennoch einen Zusammenhang zwischen der Beurteilung der Werkzeuge und der Akzeptanz der Veranstaltung gibt, wurden bivariate Korrelationen berechnet sowie eine multiple lineare Regressionsanalyse durchgeführt. Über beide Seminarformen hinweg zeigte sich, dass jedes Werkzeug für sich betrachtet einen schwachen bis mittleren Zusammenhang mit der Zufriedenheit mit dem Seminar aufweist, d. h. Personen, die mit dem Seminar insgesamt zufriedener waren, haben auch die Werkzeuge besser beurteilt. Betrachtet man die standardisierten Koeffizienten der multiplen linearen Regression, ergibt sich die Zufriedenheit mit dem Forum als einziger bedeutsamer Prädiktor (vgl. Tab. 3). 


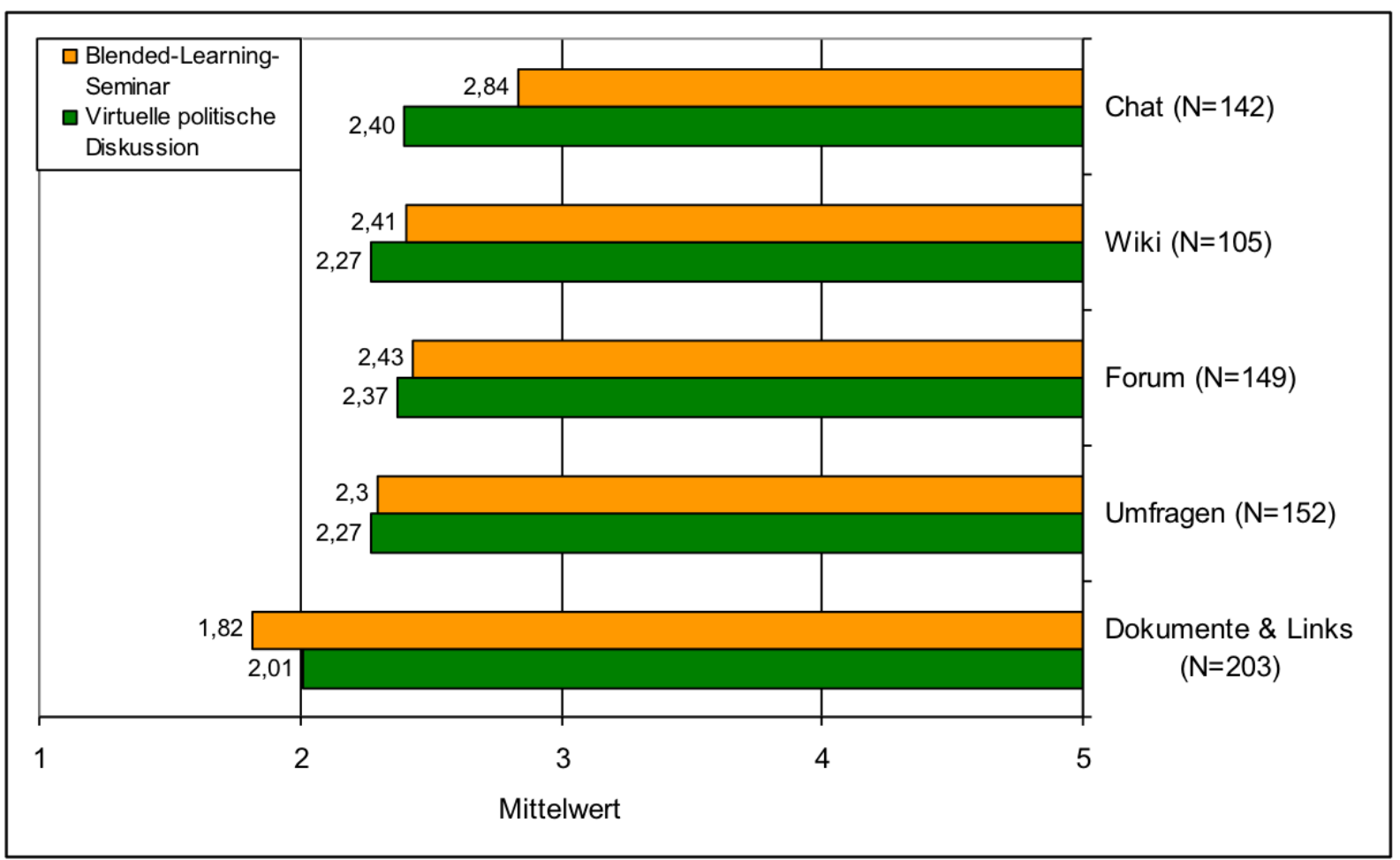

Abb. 1: Mittlere Bewertungen der Werkzeuge der Teilnehmer politischer Diskussionen und von BlendedLearning-Seminaren ( 1 = sehr gut, 5 = sehr schlecht). Die Stichprobengrösse schwankt, da nicht alle Werkzeuge in allen Seminaren angeboten, bzw. nicht von allen Teilnehmern genutzt wurden und deshalb teilweise nicht beurteilt wurden.

\begin{tabular}{|c|c|c|c|c|c|c|}
\hline \multirow{2}{*}{\multicolumn{2}{|c|}{$\begin{array}{l}\text { Gesamt } \\
\mathrm{R}=.60 \\
(\mathrm{~N}=73)\end{array}$}} & \multirow{3}{*}{$\begin{array}{l}\text { Korrelation } \\
\text { nach } \\
\text { Pearson } \\
.40\end{array}$} & \multirow{3}{*}{$\begin{array}{l}\text { Sig. } \\
\\
<.01\end{array}$} & \multirow{3}{*}{$\begin{array}{l}\text { Standardisierte } \\
\text { Koeffizienten } \\
\text { Beta } \\
.21\end{array}$} & \multirow{3}{*}{$\begin{array}{l}\mathrm{T} \\
1.56\end{array}$} & \multirow{3}{*}{$\begin{array}{l}\text { Sig. } \\
\text { n.s. }\end{array}$} \\
\hline & & & & & & \\
\hline \multirow[t]{5}{*}{1} & Dokumente \& Links & & & & & \\
\hline & $\begin{array}{l}\text { Umfragen \& Abstim- } \\
\text { mungen }\end{array}$ & .43 & $<.01$ & .17 & .746 & n.s. \\
\hline & Forum & .51 & $<.01$ & .34 & 2.86 & $<.01$ \\
\hline & Chat & .37 & $<.01$ & .11 & .94 & n.s. \\
\hline & Wiki & .27 & $<.01$ & -.06 & -.49 & n.s \\
\hline
\end{tabular}

Tab.3: Bivariate Korrelation und multiple Regression (Methode: Einschluss) der Bewertungen der Werkzeuge mit der Frage: «Wie gut hat Ihnen das Seminar insgesamt gefallen?» 
Getrennt nach Seminarform zeigt sich, dass in den rein virtuellen Diskussionsveranstaltungen der Zusammenhang zwischen der Beurteilung der Kommunikationswerkzeuge und der Zufriedenheit mit dem Seminar stärker ist als in den Blended-Learning-Veranstaltungen (messbar an den bivariaten Korrelationen, der aufgeklärten Gesamtvarianz sowie der standardisierten Koeffizienten). Die relative Bedeutung der Werkzeuge für die Zufriedenheit mit dem Seminar nimmt also im rein virtuellen Veranstaltungsformat zu, wobei besonders die Diskussionsforen von zentraler Bedeutung zu sein scheinen (vgl. Tab. 4).

\begin{tabular}{|c|c|c|c|c|c|c|}
\hline \multirow{2}{*}{\multicolumn{2}{|c|}{$\begin{array}{l}\text { Gesamt } \\
\mathrm{R}=.61 \\
(\mathrm{~N}=128)\end{array}$}} & \multirow{3}{*}{$\begin{array}{l}\text { Korrelation } \\
\text { nach } \\
\text { Pearson } \\
.25\end{array}$} & \multirow{3}{*}{$\begin{array}{l}\text { Sig. } \\
\\
<.01\end{array}$} & \multirow{3}{*}{$\begin{array}{l}\text { Standardisierte } \\
\text { Koeffizienten } \\
\text { Beta } \\
.15\end{array}$} & \multirow{3}{*}{$\begin{array}{l}T \\
2.01\end{array}$} & \multirow{3}{*}{$\begin{array}{l}\text { Sig. } \\
\\
<.05\end{array}$} \\
\hline & & & & & & \\
\hline \multirow[t]{3}{*}{1} & Dokumente \& Links & & & & & \\
\hline & Forum & .57 & $<.01$ & .46 & 5.69 & $<.01$ \\
\hline & Chat & .41 & $<.01$ & .18 & 2.30 & $<.05$ \\
\hline \multirow{2}{*}{\multicolumn{2}{|c|}{ Virtuelle politische Diskussionen }} & \multirow{2}{*}{$\begin{array}{l}\text { Korrelation } \\
\text { nach } \\
\text { Pearson }\end{array}$} & \multirow[t]{2}{*}{ Sig. } & $\begin{array}{l}\text { Standardisierte } \\
\text { Koeffizienten }\end{array}$ & & \multirow[t]{2}{*}{ Sig. } \\
\hline & & & & Beta & $\mathrm{T}$ & \\
\hline \multirow[t]{3}{*}{1} & Dokumente \& Links & .25 & $<.01$ & .13 & 1.68 & $<.10$ \\
\hline & Forum & .64 & $<.01$ & .50 & 5.69 & $<.01$ \\
\hline & Chat & .49 & $<.01$ & .24 & 2.69 & $<.01$ \\
\hline \multirow{2}{*}{\multicolumn{2}{|c|}{ Blended-Learning-Seminar }} & \multirow{2}{*}{$\begin{array}{l}\text { Korrelation } \\
\text { nach } \\
\text { Pearson }\end{array}$} & \multirow[t]{2}{*}{ Sig. } & $\begin{array}{l}\text { Standardisierte } \\
\text { Koeffizienten }\end{array}$ & & \multirow[t]{2}{*}{ Sig. } \\
\hline & & & & Beta & $\mathrm{T}$ & \\
\hline \multirow[t]{3}{*}{1} & Dokumente \& Links & .11 & $<.01$ & .03 & .16 & n.s. \\
\hline & Forum & .43 & $<.01$ & .22 & 1.26 & n.s. \\
\hline & Chat & .36 & $<.01$ & .34 & 1.93 & $<.10$ \\
\hline
\end{tabular}

Tab.4: Bivariate Korrelation und multiple Regression der Bewertungen der Werkzeuge mit der Frage: «Wie gut hat Ihnen das Seminar insgesamt gefallen?» für die Seminarformen politische Diskussion und Blended Learning (in die Regressionsmodelle wurden an dieser Stelle nur die drei am häufigsten genutzten Werkzeuge Dokumente \& Links, Forum und Chat einbezogen, wodurch sich für das Modell, das über beide Seminarformate gerechnet wurden, leichte Abweichungen zum Regressionsmodell in Tab. 3 ergeben). 
In einem zweiten Schritt wurde die Bedeutung technik-unabhängiger Merkmale für die Akzeptanz der Seminare geprüft. In die Analyse einbezogen wurde die Zufriedenheit mit dem Inhalt, mit dem Verhalten des Seminarleiters sowie mit dem Einbezug externer Experten. Auch diese Aspekte wurden durchweg positiv beurteilt, wobei die Bewertung für die Blended-Learning-Veranstaltungen hinsichtlich aller drei Merkmale besser ausfiel als für die rein virtuellen Diskussionsveranstaltungen (vgl. Abb. 2). Der Mittelwertsunterschied erwies sich dabei für alle drei Aspekte als statistisch signifikant ( $($ Inhalte $)=4.15 ; \mathrm{df}=219, \mathrm{p}<.01 ; \mathrm{t}($ Seminarleiter) $=2.84 ; \mathrm{df}=218 ; \mathrm{p}<.01 ; \mathrm{t}($ Experten $)=2.37 ; \mathrm{df}=215 ; \mathrm{p}<.05)$.

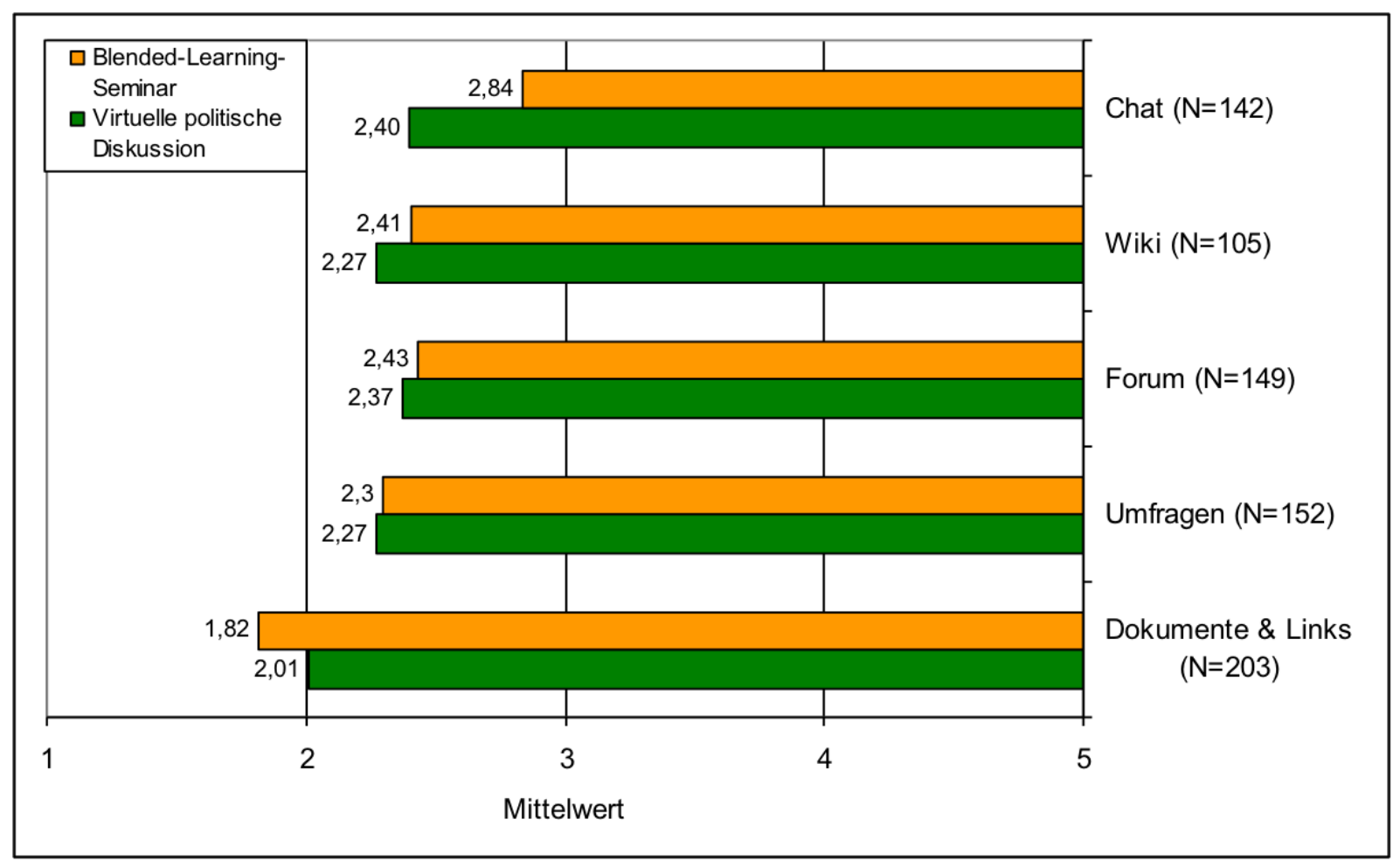

Abb. 2: Mittlere Bewertungen der Inhalte, der Seminarleitung sowie der Interaktion mit den Experten in den politischen Diskussionen und den Seminaren zum politischen Management ( 1 = sehr gut, 5 = sehr schlecht).

Um die relative Bedeutung dieser Merkmale im Vergleich zur Bedeutung der verwendeten Werkzeuge zu ermitteln, wurde im nächsten Schritt wiederum Korrelations- und Regressionsanalysen durchgeführt. Im Vergleich zur Analyse, in der nur die verwendeten Werkzeuge betrachtet wurden, zeigten sich durchweg höhere Zusammenhänge sowie eine deutlich grössere Varianzaufklärung bei der multiplen Regression (vgl. Tab. 5), was für die Bedeutung der zuletzt einbezogenen nicht- 
technischen Merkmale spricht. Von den technischen Werkzeugen trägt nur noch die Zufriedenheit mit den Diskussionsforen zur Varianzaufklärung bei, wenn die Variablen Inhalt, Seminarleitung und Experten in das Modell einbezogen werden.

\begin{tabular}{|c|c|c|c|c|c|c|}
\hline \multirow{2}{*}{\multicolumn{2}{|c|}{$\begin{array}{l}\text { Gesamt } \\
R=.79 \\
(N=127)\end{array}$}} & \multirow{3}{*}{$\begin{array}{l}\text { Korrelation } \\
\text { nach } \\
\text { Pearson } \\
.63\end{array}$} & \multirow{3}{*}{$\begin{array}{l}\text { Sig. } \\
\\
<.01\end{array}$} & \multirow{3}{*}{$\begin{array}{l}\text { Standardisierte } \\
\text { Koeffizienten } \\
\text { Beta } \\
.29\end{array}$} & \multirow{3}{*}{$\begin{array}{l}\mathrm{T} \\
3.42\end{array}$} & \multirow{3}{*}{$\begin{array}{l}\text { Sig. } \\
\\
<.01\end{array}$} \\
\hline & & & & & & \\
\hline \multirow[t]{6}{*}{1} & $\begin{array}{l}\text { Wie beurteilen Sie die Leis- } \\
\text { tungen des Seminarleiters? }\end{array}$ & & & & & \\
\hline & $\begin{array}{l}\text { Wie gut haben die bespro- } \\
\text { chenen Inhalte Ihre Erwar- } \\
\text { tungen erfüllt? }\end{array}$ & .68 & $<.01$ & .41 & 4.81 & $<.01$ \\
\hline & $\begin{array}{l}\text { Wie hat Ihnen die Interak- } \\
\text { tion mit den Experten im } \\
\text { Seminar gefallen? }\end{array}$ & .55 & $<.01$ & .03 & .03 & n.s. \\
\hline & Dokumente \& Links & .30 & $<.01$ & -.06 & -.83 & n.s. \\
\hline & Forum & .53 & $<.01$ & .19 & 2.70 & $<.01$ \\
\hline & Chat & .36 & $<.01$ & .04 & .61 & n.s. \\
\hline
\end{tabular}

Tab. 5: Bivariate Korrelation und multiple lineare Regression der Leistung des Seminarleiters, der Inhalte und der Interaktion der Experten mit der Frage: «Wie gut Ihnen das Seminar insgesamt gefallen?»

Die nach Seminarformen getrennte Analyse ergibt für die Blended-Learning-Veranstaltungen durchweg schwächere Zusammenhänge als für die virtuellen Politischen Diskussionen. In der multiplen Regression zeigt sich in den Blended-Learning-Veranstaltungen allein die Seminarleitung als signifikanter Prädiktor für die Zufriedenheit mit dem Seminar, während in den Virtuellen Politischen Diskussionen neben der Seminarleitung noch die Inhalte und die Zufriedenheit mit dem Forum als weitere Prädiktoren hinzukommen (vgl. Tab. 6). 


\begin{tabular}{|c|c|c|c|c|c|c|}
\hline \multirow{2}{*}{\multicolumn{2}{|c|}{$\begin{array}{l}\text { Virtuelle politische Diskussionen } \\
R=.79 \\
(\mathrm{~N}=92)\end{array}$}} & \multirow{3}{*}{$\begin{array}{l}\text { Korrelation } \\
\text { nach } \\
\text { Pearson } \\
.62\end{array}$} & \multirow{3}{*}{$\begin{array}{l}\text { Sig. } \\
\\
<.01\end{array}$} & \multirow{3}{*}{$\begin{array}{l}\text { Standardisierte } \\
\text { Koeffizienten } \\
\text { Beta } \\
.26\end{array}$} & \multirow{3}{*}{$\begin{array}{l}\mathrm{T} \\
2.57\end{array}$} & \multirow{3}{*}{$\begin{array}{l}\text { Sig. } \\
\\
<.05\end{array}$} \\
\hline & & & & & & \\
\hline \multirow[t]{6}{*}{1} & $\begin{array}{l}\text { Wie beurteilen Sie die Leis- } \\
\text { tungen des Seminarleiters? }\end{array}$ & & & & & \\
\hline & $\begin{array}{l}\text { Wie gut haben die bespro- } \\
\text { chenen Inhalte Ihre Erwar- } \\
\text { tungen erfüllt? }\end{array}$ & .72 & $<.01$ & .50 & 4.84 & $<.01$ \\
\hline & $\begin{array}{l}\text { Wie hat Ihnen die Interak- } \\
\text { tion mit den Experten im } \\
\text { Seminar gefallen? }\end{array}$ & .54 & $<.01$ & -.10 & -1.02 & n.s. \\
\hline & Dokumente \& Links & .31 & $<.01$ & -.04 & -.45 & n.s. \\
\hline & Forum & .60 & $<.01$ & .29 & 2.69 & $<.01$ \\
\hline & Chat & .43 & $<.01$ & .08 & .97 & n.s. \\
\hline \multirow{2}{*}{\multicolumn{2}{|c|}{ Blended Learning }} & \multirow{2}{*}{$\begin{array}{l}\text { Korrelation } \\
\text { nach } \\
\text { Pearson }\end{array}$} & \multirow[t]{2}{*}{ Sig. } & $\begin{array}{l}\text { Standardisierte } \\
\text { Koeffizienten }\end{array}$ & & \multirow[t]{2}{*}{ Sig. } \\
\hline & & & & Beta & $\mathrm{T}$ & \\
\hline \multirow[t]{6}{*}{1} & $\begin{array}{l}\text { Wie beurteilen Sie die Leis- } \\
\text { tungen des Seminarleiters? }\end{array}$ & .58 & $<.01$ & .39 & 2.34 & $<.01$ \\
\hline & $\begin{array}{l}\text { Wie gut haben die bespro- } \\
\text { chenen Inhalte Ihre Erwar- } \\
\text { tungen erfüllt? }\end{array}$ & .34 & $<.05$ & .07 & .42 & n.s. \\
\hline & $\begin{array}{l}\text { Wie hat Ihnen die Interak- } \\
\text { tion mit den Experten im } \\
\text { Seminar gefallen? }\end{array}$ & .47 & $<.01$ & .18 & 1.07 & n.s. \\
\hline & Dokumente \& Links & .11 & n.s. & -.08 & -.58 & n.s. \\
\hline & Forum & .36 & $<.05$ & .13 & .82 & n.s. \\
\hline & Chat & .43 & $<.01$ & .22 & 1.31 & n.s. \\
\hline
\end{tabular}

Tab. 6: Bivariate Korrelation und multiple lineare Regression der Leistung des Seminarleiters, der Inhalte und der Interaktion der Experten mit der Frage: «Wie gut Ihnen das Seminar insgesamt gefallen?» (getrennt nach Seminarform) 


\section{Diskussion}

Die Analyse verschiedener Seminarformate aus Nutzersicht erlaubt verschiedene Schlussfolgerungen bezüglich der Perspektiven des Web 2.0 für die Politische Bildung. Zunächst belegt die überwiegend positive Bewertung beider Kursformate die grundsätzliche Eignung internetbasierter Lern- und Diskussionsangebote für die Politische Bildung. Von zentraler Bedeutung für die Akzeptanz der Nutzer scheinen dabei die diskursiven Werkzeuge eines solchen Angebots zu sein, und dies umso mehr, wenn es keine Möglichkeit der direkten Face-to-Face Interaktion gibt. Dies spiegelt sich auch in den offenen Antworten der Teilnehmer. Insbesondere die Diskussion im Forum wurde von den Teilnehmern der virtuellen Veranstaltungen häufig und ausführlich kommentiert (allein auf das Forum bezogen sich 35 von insgesamt 70 Kommentaren), während die Teilnehmer der Blended-LearningVeranstaltungen hierzu so gut wie keine Anmerkungen machten.

Die Kommentare der Teilnehmer liefern dabei verschiedene Anhaltspunkte zur Interpretation der gefundenen Zusammenhänge. So bemängelten die Teilnehmer einerseits die unübersichtliche Fülle und mangelnde Kohärenz der Beiträge in den Foren. Darüber hinaus wurde auch die inhaltliche Qualität der Teilnehmerbeiträge kritisiert, die als oberflächlich und wenig fundiert sowie in einigen Fällen auch als unsachlich und wenig tolerant für abweichende Meinungen wahrgenommen wurden. Zur Verbesserung der Diskussion in den Foren wünschten sich die Befragten vor allem eine stärkere Moderation, mehr sachbezogenen Input durch den Seminarleiter und eine strengere Überwachung der Einhaltung von Regeln guter Kommunikation (Netiquette).

Hier zeigt sich also, dass der Einsatz virtueller Kommunikationswerkzeuge in der Politischen Bildung durchaus Probleme aufwirft, die auch bereits in anderen Studien (z. B. Jelich, 2003, s. o.) dokumentiert wurden. Diese können jedoch aus Sicht der Teilnehmer durch eine gute Moderation aufgefangen oder zumindest deutlich reduziert werden. Damit unterstreichen die Antworten der Teilnehmer ein weiteres Ergebnis der Analyse, nämlich, dass im Bereich der Politischen Bildung die Verengung des Blicks auf die technischen Werkzeuge des Internet zu kurz greift. So erwiesen sich in der regressionsanalytischen Betrachtung die nicht-technischen Faktoren wie Inhalte und Seminarleitung als deutlich bedeutsamer für die Akzeptanz der Veranstaltungen als die Zufriedenheit mit den meisten technischen Werkzeugen.

Erwähnenswert ist weiterhin, dass insgesamt die Blended-Learning-Veranstaltungen eine noch bessere Akzeptanz fanden als die ausschliesslich virtuellen politischen Diskussionen. Da sich die beiden Seminarformate grundsätzlich stark unterscheiden, spielt hier sicherlich neben dem Format (rein virtuell vs. blended learning) eine Reihe von Faktoren eine Rolle, die in der vorliegenden Untersuchung nicht betrachtet wurden (z. B. Unterschiede hinsichtlich der Lernziele, der Dauer, 
der Gruppengrösse und der angesprochenen Zielgruppe). Die Ergebnisse zeigen dennoch, dass die insgesamt höhere Akzeptanz der Blended-Learning-Veranstaltungen weniger mit der Zufriedenheit mit den Werkzeugen zusammenzuhängen scheint, denn hier bestanden keine statistisch nachweisbaren Unterschiede zwischen den Teilnehmern von Blended-Learning-Veranstaltungen und von rein virtuellen Seminaren. Hinsichtlich der Inhalte, der Qualität der Seminarleitung und der Einbindung von Experten wurden sie dagegen signifikant besser bewertet. Möglicherweise tragen die Präsenzphasen der Blended-Learning-Seminare dazu bei, ein vertrauensvolles Verhältnis zum Seminarleiter und zu den eingeladenen Experten zu etablieren und die Inhalte der Seminare besser zu kommunizieren.

Einen weiteren Hinweis auf einen möglichen Vorteil von Blended-Learning-Veranstaltungen geben wiederum die Antworten auf die offene Frage zur Akzeptanz der Seminare. So wurde von zahlreichen Befragungsteilnehmern geäussert, dass sie sich gewünscht hätten, häufiger kooperativ zu arbeiten und auch engere Kontakte zu den anderen Seminarteilnehmern zu knüpfen bzw. diese noch besser persönlich kennenzulernen. Insgesamt bestand bei vielen Teilnehmern der Wunsch nach Vernetzung mit Gleichgesinnten auch über das besuchte Seminar hinaus, der insbesondere in den rein virtuellen Veranstaltungen häufig unerfüllt blieb. BlendedLearning-Veranstaltungen haben hier Vorteile, obwohl sich auch hier die Teilnehmer noch eine intensivere und dauerhaftere Vernetzung gewünscht hätten.

\section{Fazit}

Angebotsanalyse und Nutzerbefragung ergänzen sich zu einem komplexen Bild der Rolle des Web 2.0 in der Politischen Bildung. Zunächst machen beide Analysen deutlich, dass Web-2.0-Technologien bereits begonnen haben, ihr Potenzial in der Politischen Bildung zu entfalten. Dies belegt besonders die Angebotsanalyse, die zeigen konnte, dass in den letzten Jahren eine Vielzahl neuer Plattformen für den politischen Meinungsaustausch entstanden ist, von denen sich viele einer regen Teilnahme erfreuen. Auch die insgesamt recht positive Bewertung der verschiedenen E-Learning-Werkzeuge wie auch der rein virtuellen Diskussion durch die Teilnehmer in der durchgeführten Nutzerbefragung unterstützt das Potenzial des Web 2.0 für die Politische Bildung. Die Ergebnisse von Angebotsanalyse und Nutzerberfragung machen jedoch auch offensichtlich, dass Politische Bildung mehr bedeutet als eine Plattform für die Diskussion politischer Themen im Internet oder zur Vernetzung politisch Interessierter bereitzustellen, und zwar sowohl aus Sicht der Anbieter wie auch aus Sicht der Nutzer. Aus Sicht der Anbieter zeigt sich, dass gerade die traditionellen Anbieter Politischer Bildung sich bisher noch mit der Adaption von Web-2.0-Technologien schwer tun. Zu einem vergleichbaren Ergebnis kam auch eine von Boeser et al. im Jahr 2007 in Bayern durchgeführte Umfrage unter 200 traditionellen Präsenz-Institutionen, die Politische Bildung an- 
bieten (Volkshochschulen, politische Stiftungen, Zentralen für Politische Bildung). Die Autoren fanden, dass gerade einmal 13,1\% der befragten Institutionen virtuelle Angebote machen (Boeser et al., 2007). Wiederholt wurde festgestellt, dass sich die traditionellen Bildungsträger gegenüber dem Internet als Lernmedium durch eine grundlegende Skepsis auszeichnen. So fanden Fritz et al. (2006) in einer kombinierten Befragung von Institutionen, Erwachsenenbildnern und Teilnehmern politischer Bildungsangebote, dass $96 \%$ der befragten politischen Bildnerinnen die Überzeugung vertreten, dass mit dem Internet allein keine Politische Bildung betrieben werden könne. Auch andere Autoren weisen daraufhin, dass in der Politischen Bildung die Präsenzveranstaltung als Königsweg zur Vermittlung politischer Inhalte gesehen wird und virtuelle Angebote mit Argwohn betrachtet werden (Meyer, 2000; Rilling, 2000; Wache, 2003). Neben einer aus der Tradition der Institutionen erwachsenden Präferenz für Präsenzveranstaltungen bei den traditionellen Anbietern politischer Bildung mag ein (zunächst inhaltsleeres) Angebot wie eine Diskussions- oder Vernetzungsplattform mit dem Selbstverständnis als Vermittler politischer Bildung vermutlich auch kaum vereinbar sein, denn zu diesem Selbstverständnis gehört nicht nur die didaktische Aufbereitung und angemessene Präsentation politischer Inhalte und Themen, sondern auch der fundierte und qualitativ hochwertige Diskurs, der durch das unbetreute Angebot einer Vernetzungs- und Diskussionsplattform nicht gewährleistet werden kann. Die Nutzerbefragung zeigt, dass sich diese Einschätzung durchaus mit den Teilnehmerinteressen, bzw. Erwartungen auch an virtuelle politische Diskussionsveranstaltungen deckt. So wurde insbesondere in der rein virtuellen Diskussion häufig der Wunsch nach einem Eingreifen des Moderators laut, um die Diskussion zu strukturieren, ihr einen Fokus zu geben und die Qualität der Beiträge zu steigern.

Der Weg der traditionellen Anbieter Politischer Bildung, Mischformen zu erproben, die eine deutliche Nähe zum klassischen Präsenzangebot, bzw. zum herkömmlichen betreuten E-Learning aufweisen, mag also aus technisch-didaktischer Sicht zunächst wenig innovativ erscheinen, ist mit Blick auf die Nutzerperspektive aber durchaus gerechtfertigt. Einen Schlüssel für die Verbindung «klassischer» Angebote und dem Web 2.0 könnte das Bedürfnis der Teilnehmer nach besserer und langfristiger Vernetzung und Bildung von Interessengruppen darstellen. Eine Aufgabe der Anbieter Politischer Bildung würde dann für die Zukunft darin bestehen, Konzepte zu entwickeln, wie aus den Teilnehmern ihrer Angebote - seien es Präsenzveranstaltungen oder virtuelle Lernangebote - virtuelle Communitys von Politik-Interessierten und -Engagierten entstehen können und so ihrem Auftrag, die gesellschaftliche und politische Partizipation der Bürger zu stärken, auch mit Blick auf die Partizipationschancen im Internet gerecht zu werden. 


\section{Literatur}

Boeser, Christian et al. 2007. Politische Bildung Bayern. Projektbericht. Augsburg: Universität Augsburg.

Brombach, Guido. 2007. «Wikimethodix - Einsatz von Wikis in der (politischen) Erwachsenenbildung.»kommunikation@gesellschaft 8, 1. Internet-Dokument: http://www.soz.uni-frankfurt.de/K.G/F1_2007_Brombach.pdf [8.8.2011].

Ehlers, Ulf-Daniel. 2008. "Qualität für neue Lernkulturen des «Next Generation» E-Learning. In Online-Lernen: Handbuch für Wissneschaft und Praxis, hrsg. v. Ludwig J. Issing u. Paul Klimsa, 339-356. München: Oldenbourg Verlag.

Fritz, Karsten, Katharina Maier und Lothar Böhnisch. 2006. Politische Erwachsenenbildung: Trendbericht zur empirischen Wirklichkeit der Politischen Bildung in Deutschland. Weinheim: Juventa.

Fuchs, Christian. 2006. «eParticipation Research: A case study on political online debate in Austria.» Research Paper No 1. Salzburg: Universität Salzburg, ICT\&S Center.

Harth, Thilo. 2000. Das Internet als Herausforderung politischer Bildung. Schwalbach/Ts.: Wochenschau-Verlag.

Jelich, Franz-Joseph. 2003. "Kommunikationsraum Internet - ein Ort politischer Bildung?» Literatur- und Forschungsreport Weiterbildung 26 (1): 74-82.

Meyer, Thomas. 2000. «Internet und Politische Bildung - Zehn Thesen.»InternetDokument: http://www.bildungsforum21.de/themenportal/hintergrund_zehnthesen.html [23.11.2010].

Ott, Raphaela. 2006. «Weblogs als Medium politischer Kommunikation im Wahlkampf 2005.» In Die Massenmedien im Wahlkampf: Die Bundestagswahl 2005, hrsg. v. Christina Holtz-Bacha, 213-233. VS Verlag für Sozialwissenschaften.

Pettingill, Lindsay. 2008. «Engagement 2.0? How the new digital media can invigorate civic engagement.» gnovis journal 8 (3): 155-161. Internet-Dokument: http://www.gnovisjournal.org/files/Engagement-2-0.pdf [8.8.2011].

Raynes-Goldie, Kate und Luke Walker. 2008. «Our Space: Online civic engagement tools for youth.» In Civic Life Online: Learning how digital media can engage youth, hrsg. v. W. L. Bennett, 161-188. The John D. and Catherine T. MacArthur Foundation Series on Digital Media and Learning. Cambridge, MA: The MIT Press.

Rheingold, Howard. 2008. "Using participatory media and public voice to encourage civic engagement.»In Civic Life Online: Learning how digital media can engage youth, hrsg. v. W. L. Bennett, 97-118. The John D. and Catherine T. MacArthur Foundation Series on Digital Media and Learning. Cambridge, MA: The MIT Press.

Rilling, Rainer. 1999. "Elektronische Kommunikations- und Informationsnetze Chancen und Aufgaben für die Politische Bildung.» In Jahrbuch Arbeit, Bil- 
dung, Kultur, hrsg. v. Forschungsinstitut für Arbeiterbildung, Bd. 17, 69-77. Recklinghausen.

Rilling, Rainer. 2000. «Internet und Politische Bildung.» Utopie kreativ 121/122: 1080-1089.

Rosa, Lisa. 2006. «Das Weblog als Instrument der Politischen Bildung.»Internet-Dokument: http://static.twoday.net/LisaRosa/files/Das-Weblog-als-Instrumentder-politischen-Bildung.pdf [8.8.2011].

Ruprecht, Gisela. 2002. Politische Bildung im Internet. Schwalbach: WochenschauVerlag.

Schulz-Zander, Renate und Gerhard Tulodziecki. 2008. «Pädagogische Grundlagen des Online-Lernens.» In Online-Lernen: Handbuch für Wissneschaft und Praxis, hrsg. v. Ludwig J. Issing u. Paul Klimsa, 35-45. München: Oldenbourg Verlag.

Selwyn, Neil. 2007. Report 3 Update: Citizenship, technology and learning - a review of recent literature. Futurelab Series. London: Futurelab. Internet-Dokument: http://archive.futurelab.org.uk/resources/documents/lit_reviews/Citizenship_Review_update.pdf [8.8.2011].

Wache, Michael. 2003. «E-Learning - Bildung im digitalen Zeitalter.» Internet-Dokument: http://www.bpb.de/files/FWOFK9.pdf [8.8.2011]. 